\title{
BMJ Open Cost-efficiency of specialist inpatient rehabilitation for working-aged adults with complex neurological disabilities: a multicentre cohort analysis of a national clinical data set
}

\author{
Lynne Turner-Stokes, ${ }^{1,2}$ Heather Williams, ${ }^{2}$ Alan Bill, ${ }^{2}$ Paul Bassett, ${ }^{3}$ \\ Keith Sephton ${ }^{2}$
}

To cite: Turner-Stokes $L$, Williams $\mathrm{H}$, Bill A, et al. Costefficiency of specialist inpatient rehabilitation for working-aged adults with complex neurological disabilities: a multicentre cohort analysis of a national clinical data set. BMJ Open 2016;6:e010238.

doi:10.1136/bmjopen-2015010238

- Prepublication history for this paper is available online. To view these files please visit the journal online (http://dx.doi.org/10.1136/ bmjopen-2015-010238).

Received 10 October 2015 Revised 23 November 2015 Accepted 9 December 2015

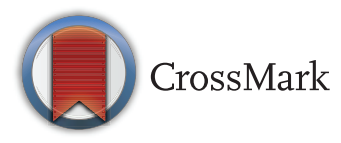

${ }^{1}$ Faculty of Life Sciences and Medicine, Department of Palliative Care, Policy and Rehabilitation, King's College London, London, UK

${ }^{2}$ Regional/Hyper-acute Rehabilitation Unit, Northwick Park Hospital, UK

${ }^{3}$ Statsconsultancy Ltd,

London, UK

Correspondence to

Professor Lynne

Turner-Stokes:

lynne.turner-stokes@dial.

pipex.com

\section{ABSTRACT}

Objectives: To evaluate functional outcomes, care needs and cost-efficiency of specialist rehabilitation for a multicentre cohort of inpatients with complex neurological disability, comparing different diagnostic groups across 3 levels of dependency.

Design: A multicentre cohort analysis of prospectively collected clinical data from the UK Rehabilitation Outcomes Collaborative (UKROC) national clinical database, 2010-2015.

Setting: All 62 specialist (levels 1 and 2) rehabilitation services in England.

Participants: Working-aged adults (16-65 years) with complex neurological disability. Inclusion criteria: all episodes with length of stay (LOS) 8-400 days and complete outcome measures recorded on admission and discharge. Total $\mathrm{N}=5739$ : acquired brain injury $\mathrm{n}=4182(73 \%)$; spinal cord injury $\mathrm{n}=506(9 \%)$; peripheral neurological conditions $n=282(5 \%)$; progressive conditions $n=769(13 \%)$.

Intervention: Specialist inpatient multidisciplinary rehabilitation.

Outcome measures: Dependency and care costs: Northwick Park Dependency Scale/Care Needs Assessment (NPDS/NPCNA). Functional independence: UK Functional Assessment Measure (UK Functional Independence Measure (FIM)+FAM). Cost-efficiency: (1) time taken to offset rehabilitation costs by savings in NPCNA-estimated costs of ongoing care, (2) FIM efficiency (FIM gain/LOS days), (3) FIM+FAM efficiency (FIM+FAM gain/LOS days). Patients were analysed in 3 groups of dependency.

Results: Mean LOS 90.1 (SD 66) days. All groups showed significant reduction in dependency between admission and discharge on all measures (paired $t$ tests: $p<0.001)$. Mean reduction in 'weekly care costs' was greatest in the high-dependency group at $£ 760$ / week (95\% Cl 726 to 794)), compared with the medium-dependency (£408/week (95\% Cl 370 to 445)), and low-dependency (£130/week (95\% Cl 82 to 178)), groups. Despite longer LOS, time taken to offset the cost of rehabilitation was $14.2(95 \% \mathrm{Cl} 9.9$ to 18.8) months in the high-dependency group,

\section{Strengths and limitations of this study}

- A large 5-year national consecutive cohort analysis representing all specialist (levels 1 and 2) rehabilitation units in England

- Prospective routinely collected data are reflective of real clinical practice.

- Different methods for evaluation of costefficiency are compared in the same data set.

- Owing to evolution of reporting requirements over the data collection period, the outcomes of interest were collected in less than $50 \%$ of the full rehabilitation data set, so selection bias cannot be excluded.

- This highly selected group of patients with complex needs is atypical in comparison to populations described in published analyses from other large data sets, but has potential relevance for other health systems that provide tertiary specialist rehabilitation services.

compared with $22.3(95 \% \mathrm{Cl} 16.9$ to 29.2$)$ months (medium dependency), and 27.7 (95\% Cl 15.9 to 39.7) months (low dependency). FIM efficiency appeared greatest in medium-dependency patients (0.54), compared with the low-dependency $(0.37)$ and high-dependency (0.38) groups. Broadly similar patterns were seen across all 4 diagnostic groups.

Conclusions: Specialist rehabilitation can be highly cost-efficient for all neurological conditions, producing substantial savings in ongoing care costs, especially in high-dependency patients.

\section{INTRODUCTION}

Over 1 million people in the UK ( $2 \%$ of the population) have a disabling neurological condition, of which 350000 require help for most of their daily activities and it is estimated that 850000 people care for someone 
with a neurological condition. ${ }^{1}$ By improving independence and autonomy, rehabilitation has the potential to reduce the needs for care and thus relieve the burden and costs of care, both for family and society. Although there is a growing body of trial-based evidence for the effectiveness of rehabilitation in a variety of neurological conditions, ${ }^{2}{ }^{3}$ there are other important questions that require a practice-based approach to determine what works best for which patients and what approaches represent value for money in the context of real-life clinical practice. $^{45}$

Much of the evidence for effectiveness of rehabilitation comes from the arenas of stroke and care of older people. To date, there has been relatively little focus on younger (ie, working aged) adults with complex disability following neurological illness or injury. Specialist rehabilitation is increasingly recognised as an essential component of healthcare for this group of patients. ${ }^{6}$ However, it can be a costly intervention and systematic evaluation is required to demonstrate that programmes are both effective and cost-efficient. Porter and Teisberg ${ }^{7}$ introduced the concept of 'value-based healthcare', where the goal is not necessarily to minimise costs but to maximise 'value', defined as 'patient outcomes divided by costs'.

The Functional Independence Measure (FIM) is the most widely used standardised outcome measure for rehabilitation in the world. Established large rehabilitation data sets in the USA and Australia rely on the FIM, not only as a measure of functional gains during rehabilitation, but as a casemix tool and a measure of cost-efficiency. In the absence of direct costing data, the 'FIM-efficiency index' (FIM gain/length of stay (LOS)) is often used as a proxy for cost-efficiency. ${ }^{8-13}$ However, such estimations have a number of weaknesses:

1. They assume linearity of change and equal weighting of items to the prediction of overall cost of care, which is not necessarily the case.

2. They are frequently confounded by floor and ceiling effects. $^{14}$

3. The FIM is largely focused on physical disability, which limits its use in the context of complex neurological disability, where cognitive and psychosocial problems are often the principal limiting factors.

The UK National Health Service (NHS) provides one of the most comprehensive health and social service systems in the world ${ }^{15}$ and demands a somewhat different approach.

- Rehabilitation services are planned and provided in coordinated regional networks over a relatively small geographical area. Local general (level 3) rehabilitation services provide for the majority of patients, but a smaller number are referred to specialist (level 1 or 2) services, which take a selected population of mainly younger adults with complex needs for rehabilitation that are beyond the scope of their local rehabilitation services. ${ }^{16}$

- The statutory commitment to life-long provision of care supports longer periods of rehabilitation in these specialist services, provided that this can be demonstrated to produce meaningful cost-benefits through gains in wider independence and reduction of long-term care needs.

Since 2010, the national UK Rehabilitation Outcomes Collaborative (UKROC) database has collated episode data for all inpatients admitted to specialist rehabilitation services (levels 1 and 2) in England, providing national benchmarking on quality, outcomes and costefficiency of rehabilitation. Within the UKROC data set, functional gain is evaluated using the UK Functional Assessment Measure (UK FIM+FAM) ${ }^{17}{ }^{18}$ which extends the FIM to provide greater coverage of cognitive and psychosocial function. Cost-efficiency is computed in terms of the length of time taken to offset the initial costs of rehabilitation through savings in the ongoing costs of community care as estimated by the Northwick Park Dependency Care Needs Assessment. ${ }^{19} 20$

A previously published single-centre analysis using these indices demonstrated the cost-efficiency of rehabilitation for younger adults with complex needs following acquired brain injury (ABI),${ }^{21}$ and showed that longer lengths of stay can provide value for money by reducing ongoing care costs. ${ }^{22}$ The cost-benefits were particularly marked for highly dependent patients, while 'FIM efficiency' appeared to be greatest for the mediumdependency group. This finding was important as highly dependent patients may be denied rehabilitation in other healthcare systems on the basis that they are costly to care for and not expected to make significant gains on the FIM. ${ }^{21}$

The objective of this article is to present the first national cohort analysis of the UKROC database to describe functional outcome, change in care needs and cost-efficiency following specialist rehabilitation for working-aged adults with complex disability arising from neurological conditions. In particular, we wished to determine whether the single-centre findings above were reproducible across multiple centres and across a wider range of neurological conditions.

Specific research questions were:

1. What types of functional gain are made during rehabilitation by patients with different neurological conditions?

2. Can longer lengths of stay for highly dependent patients be justified by savings in ongoing care costs?

3. Are there important differences in outcome and costefficiency across different neurological conditions and for different levels of patient dependency that service planners should be aware of?

\section{METHODS}

Design

A large 5-year multicentre national cohort analysis of prospectively collected clinical data from the UKROC national clinical database 2010-2015. Participants were working-aged adults (aged 16-65) with complex 
neurological disability undergoing specialist inpatient rehabilitation in England.

\section{Setting and data source}

In England, level 1 rehabilitation units are tertiary services providing for a regionally based catchment population of 3-5million and taking a highly selected caseload of patients with very complex needs. They are subdivided by casemix into hyperacute, 1a (physical disability), 1c (cognitive behavioural) and 1b (mixed) services. Level 2 services take a mixed caseload providing for a more local population, divided into 2a (supradistrict) and 2b (local district) specialist rehabilitation services. The data reporting requirements have evolved over time and vary somewhat between the different levels of service.

The UKROC database was established in 2009 through funding a programme grant from the UK National Institute for Health Research (NIHR), ${ }^{23}$ but now provides the national commissioning data set for NHS England. The database collates de-identified data, which are uploaded at monthly intervals and stored on a secured NHS server held at Northwick Park Hospital. It is overseen by a steering group of the British Society of Rehabilitation Medicine.

The data set comprises sociodemographic and process data (waiting times, discharge destination, etc) as well as clinical information on rehabilitation needs, inputs and outcomes. Full details may be found on the UKROC website http://www.csi.kcl.ac.uk/ukroc.html.

- Data collection started formally in April 2010. Reporting was initially voluntary and contributing centres could report any one of three approved outcome measures, the Barthel Index (BI), the FIM or UK FIM+FAM.

- Since April 2012, levels 1 and 2a services are commissioned centrally by NHS England and are required to report the full UKROC data set for all admitted episodes, including the UK FIM+FAM as the principal outcome measure.

- Reporting of the Northwick Park Dependency Scale and Care Needs Assessment as a measure of costefficiency was optional until April 2013, but is now a requirement for national benchmarking for these levels 1 and 2a services.

- Locally commissioned level 2b (local district) services may still report only lower level data such as the BI or FIM.

\section{Measurements}

The UK FIM+FAM is a global measure of disability. ${ }^{17}{ }^{18}$ It includes the 18-item FIM (V.4) and adds a further 12 items, mainly addressing psychosocial function giving a total of 30 items (16 motor and 14 cognitive items). Each item is scored on a seven-point ordinal scale from 1 (total dependence) to 7 (complete independence). Further details are published elsewhere. ${ }^{17} 18$

The Northwick Park Dependency Score (NPDS) is an ordinal scale of dependency on nursing staff time (number of helpers and time taken to assist with each task) designed to assess needs for care and nursing in clinical rehabilitation settings. ${ }^{19}$ It comprises a 16 -item scale of basic care needs (range 0-65) and a 7-item scale of special nursing needs (range 0-35) - total range 0100 . It is shown to be a valid and reliable measure of needs for care and nursing in rehabilitation settings. ${ }^{24}$ It supports categorisation of patients into three dependency groups based on their admission NPDS: $:^{21}$

- Low dependency (NPDS <10): patients are largely independent for basic self-care,

- Medium (NPDS 10-24): patients generally require help from one person for most self-care tasks,

- High (NPDS $\geq 25$ ): patients require help from two or more persons for most care tasks and often also have special nursing needs.

The NPDS also translates via a computerised algorithm to the Northwick Park Care Needs Assessment (NPCNA) ${ }^{20}$ which estimates the total care hours per week and the approximate weekly cost of care (£/week) in the community, based on the UK care agency costs. The NPCNA provides a generic assessment of care needs, regardless of who provides and pays for them. The estimated cost of care is therefore independent of individual circumstances or local policy for the provision continuing care, which varies widely across the UK. The algorithm is embedded within the UKROC software and generates this information automatically.

Although there is no formal accreditation process for use of the UK FIM+FAM and NPDS, the attendance of UK FIM+FAM training by at least a core team of staff is requirement for UKROC registration. All units that are registered with UKROC have access to the national training and update workshops, as well as free telephone support.

\section{Cost-efficiency of rehabilitation}

Within the UKROC data set, the cost-efficiency is calculated as the time taken to offset the cost of rehabilitation by the resulting savings in the cost of ongoing care in the community. This is calculated from the 'episode cost of rehabilitation' divided by 'reduction in weekly cost of care' from admission to discharge, as estimated by the NPCNA. The episode cost was calculated per patient as 'bed-day cost $\times$ LOS'. The cost per bed-day was calculated on updated data from our previously published costanalysis. ${ }^{25}$ We used mean per diem costs for the different levels of service as follows: 1 hyperacute: $£ 670$, 1a: $£ 540,1$ b: $£ 483,1$ c: $£ 634$, 2a: 452, 2b: £418. For comparison with other series, we also report FIM efficiency, calculated at individual patient level as change in total FIM score/LOS in days. FIM+FAM efficiency is calculated as change in total UK FIM+FAM score/LOS in days.

\section{Valid LOS}

In order to identify plausible admissions for rehabilitation (as opposed to brief inpatient assessment or for long-term care) we selected patients with LOS between 8 
and 400 days. Other cohort studies have used similar cut-off points, ${ }^{26}$ although the exact time frames may vary according with local practice. In this cohort, we excluded patients staying for 1 week or less as they would not meet even the lowest time-thresholds for repeat assessment. The NHS England service specification for rehabilitation stipulates a maximum programme length of 180 days with a trim point of 14 days (ie, 194 days in total). Subject to approval, extension for a second period may be granted in some cases if it can be justified on the grounds of anticipated functional gain and cost-efficiency, bringing the total allowed LOS to 388 days. Allowing for possible short delays in discharge at the end of programme, we therefore set 400 days as the ceiling for a plausible LOS for rehabilitation.

\section{Data extraction}

De-identified data were extracted for all recorded inpatient episodes for adults aged 16-65 years admitted to level 1 or 2 specialist rehabilitation service and discharged during the 5-year period between 1.4.2010 and 31.3.15, if they had:

A. A neurological condition recorded in the diagnostic category;

B. A LOS 8-400 days;

C. Valid UK FIM+FAM and NPDS ratings completed both within 10 days of admission and within the last week before discharge.

Data were collated in MS Excel and transferred to SPSS V.22 for analysis.

\section{Data handling and analysis}

Because data reporting was initially voluntary, missing data were expected. No data were imputed for missing values. There is continued debate about whether to use parametric or non-parametric statistics for this type of data. In this analysis, given the large size of the data set and long ordinal nature of the measures (ie, many possible data points), we have elected to describe and analyse the data using parametric statistics—although non-parametric analysis gave very similar results and is available from the authors if required.

- Ninety-five per cent CIs were calculated and multiple comparisons made using bootstrapping with samples of $n=1000$, to minimise the effect of any skewed data.

- Paired t tests were used to compare significant differences between admission and discharge.

- One-way analyses of variances (ANOVAs) with bootstrapped post hoc analysis and Bonferroni correction to correct for multiple tests were used to compare differences for diagnostic groups and for different levels of dependency. Key results from post hoc analyses are summarised in the text, but not given in tables. Further details are available on request from the corresponding author.

In this non-interventional observational study, size was not predetermined but dictated by the accruals to the national data set over the 5-year period that met the inclusion criteria. Because the data set was dominated by patients with $\mathrm{ABI}$, analysis was also undertaken separately for each diagnostic group.

\section{RESULTS}

Figure 1 illustrates the data extraction process. From a total of 13004 registered episodes for adults aged 16-65 with a neurological condition, 12256 had a LOS between 8 and 400 days representing the data set of adults admitted for rehabilitation. Of these, 5739 (47\%) had a valid NPDS and FIM+FAM on both admission and discharge and were included in the analysed sample.

A total of 62 rehabilitation units (15 level 1, 15 level 2a and 32 level $2 \mathrm{~b}$ services) provided data, with good representation across all four health regions in England.

Demographics are given in table 1. Because the sample comprised less than $50 \%$ of the total rehabilitation data set, demographics were compared for the analysed and the total sample. No significant differences were found.

The study sample comprised approximately 3:2 males: females, with a mean age at admission of $47.3(\mathrm{SD}=12.6)$
Figure 1 The data extraction process to derive the data set used for analysis. FIM, Functional Independence Measure; FIM +FAM, UK Functional Assessment Measure; NPDS, Northwick Park Dependency Score.

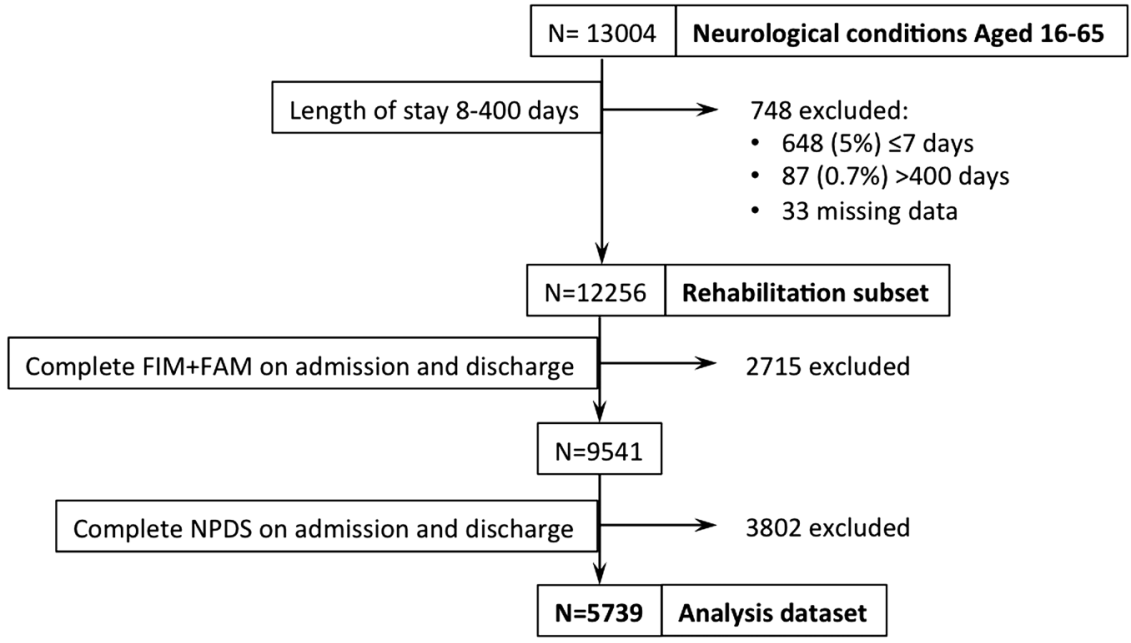




\begin{tabular}{|c|c|c|c|c|c|c|c|}
\hline Parameter & Missing $n=$ & $\begin{array}{l}\text { All } \\
N=5739\end{array}$ & $\begin{array}{l}A B I \\
N=4182(73 \%)\end{array}$ & $\begin{array}{l}\mathrm{SCl} \\
\mathrm{N}=506(9 \%)\end{array}$ & $\begin{array}{l}\text { Peripheral } \\
\mathrm{N}=282(5 \%)\end{array}$ & $\begin{array}{l}\text { Progressive } \\
\mathrm{N}=769(13 \%)\end{array}$ & $\begin{array}{l}\text { Full data set } \\
N=12256^{*}\end{array}$ \\
\hline \multicolumn{8}{|l|}{ Age } \\
\hline Mean (SD) & 0 & $47.3(12.6)$ & $46.8(12.8)$ & $49.3(12.7)$ & $47.8(12.8)$ & $48.6(10.8)$ & $47.0(12.8)$ \\
\hline M:F ratio (\%) & 4 & $59 / 41 \%$ & $62 / 38 \%$ & $59 / 41 \%$ & $55 / 45 \%$ & $40 / 60 \%$ & $60 / 40 \%$ \\
\hline \multicolumn{8}{|l|}{ Time since onset (days) } \\
\hline Mean (SD) & & 657 (2093) & 237 (1196) & $660(2763)$ & 139 (359) & 3223 (3576) & $691(2273)$ \\
\hline Median (IQR) & & $59(29-137)$ & $54(28-104)$ & $48(25-136)$ & $60(30-11)$ & $2326(90-5031)$ & $57(28-133)$ \\
\hline \multicolumn{8}{|l|}{ Length of stay (days) } \\
\hline Mean (SD) days & 0 & $90.1(65.5)$ & $90.7(67.4)$ & $72.8(58.5)$ & $79.9(60.6)$ & $56.3(60.0)$ & $79.2(67.3)$ \\
\hline \multicolumn{8}{|l|}{ Cost of episode } \\
\hline Mean (SD) & 0 & $£ 39381$ (£32 235) & $£ 43053$ (£33 473) & $£ 32813$ (£26 519) & $£ 36631$ (£31.357) & $£ 24739$ (£22 857) & $£ 37158(£ 33121)$ \\
\hline \multicolumn{8}{|c|}{ Diagnostic subcategories n (\%) } \\
\hline Trauma & & 1259 (21.9) & $1127(26.9)$ & $125(24.7)$ & $7(2.5)$ & & $2769(22.6)$ \\
\hline Vascular & & $2048(35.7)$ & $1979(47.7)$ & $49(9.7)$ & $20(7.1)$ & & $4299(35.1)$ \\
\hline Inflammatory & & $448(7.8)$ & $175(4.2)$ & $109(21.5)$ & $164(58.2)$ & & $950(7.7)$ \\
\hline Tumour & & $347(6.0)$ & $268(6.4)$ & 79 (15.6) & - & & $705(5.8)$ \\
\hline Other & & 934 (16.3) & $595(14.3)$ & $140(27.7)$ & $89(31.6)$ & $110(14.3)$ & 1864 (15.3) \\
\hline Multiple sclerosis & & $636(11.1)$ & & & & $636(82.7)$ & $1323(10.8)$ \\
\hline Motor neurone disease & & $7(0.1)$ & & & & $7(0.9)$ & $16(0.1)$ \\
\hline Parkinson's disease & & $13(0.2)$ & & & & $13(1.7)$ & $23(0.2)$ \\
\hline Missing & & $47(0.8)$ & $38(0.9)$ & $4(0.8)$ & $2(0.7)$ & & $307(2.5)$ \\
\hline
\end{tabular}

No significant differences were seen between the demographics of the analysis dataset and the full data set.

${ }^{*} \mathrm{~N}=12256$ is made up of $9000(73 \%) \mathrm{ABI}, 977(8 \%) \mathrm{SCl}, 642(5 \%)$ peripheral and 1637 (13\%) progressive conditions.

$\mathrm{ABI}$, acquired brain injury; $\mathrm{F}$, female; $\mathrm{M}$, male; $\mathrm{SCl}$, spinal cord injury. 
years. The mean rehabilitation LOS was $90.1 \quad(\mathrm{SD}=65.5)$ days. Nearly three-quarters of the sample $(73 \%)$ had $\mathrm{ABI}$, the remainder having spinal cord injuries (SCIs; $9 \%$ ), peripheral neurological conditions, for example, Guillain-Barré syndrome $(5 \%)$ and progressive conditions $(13 \%)$. Table 1 shows the demographics for these diagnostic groups and shows the breakdown of aetiological causes within each category. As the time between onset and admission ('time since onset') was very highly skewed, the median and IQR is given as well as the mean (SD). Excluding the progressive conditions, the mean time since onset for ABI, SCI and peripheral neurological conditions was 9.0 months (SD 46.5).

One-way ANOVA tests confirmed significant differences in LOS and episode costs $(p<0.001)$ between the different diagnostic groups. Patients with ABI stayed longest (mean 90 days) with the highest episode costs (mean approximately $£ 43000$ ), while those with progressive conditions stayed the shortest (mean 56 days) and corresponding lower episode costs (mean approximately $£ 25000)$.

\section{Dependency and functional outcomes}

Table 2 summarises the overall dependency and functional outcome scores for the sample, together with costefficiency. Between admission and discharge there was highly significant increase in all parameters of functional independence $(\mathrm{FIM}+\mathrm{FAM}$; $\mathrm{p}<0.001)$, with corresponding reduction in all parameters of dependency (NPDS/ NPCNA; $p<0.001)$. The mean total FIM+FAM gain was 35.5 and the mean individually calculated FIM+FAM efficiency/week was 0.67 (95\% CI 0.64 to 0.69 ). The mean total cost of the rehabilitation programme was $£ 39381$ and mean savings in ongoing cost of care in the community was $£ 496 /$ week. The mean time taken to offset the initial costs of rehabilitation was 17.9 months $(95 \%$ CI 14.5 to 21.4$)$.

\section{Differences between diagnostic groups}

The UKROC software generates 'FAM splats' in the form of radar charts which provide an 'at a glance' view of the disability profile and patterns of change during rehabilitation for the 30 FIM+FAM items. Figure 2 shows the composite FAM splats based on median item scores at admission and discharge for the four main diagnostic groups. They illustrate the clinical value of recording change in psychosocial, as well as physical function, which would not be detected by changes in the FIM items alone.

The differences in functional outcome across the diagnostic groups are summarised in table 3. On admission, FIM+FAM motor scores were broadly similar across all

Table 2 Overall dependency and functional outcome scores on admission and discharge $(n=5739)$

\begin{tabular}{|c|c|c|c|c|c|c|}
\hline & $\begin{array}{l}\text { Admission } \\
\text { Mean (SD) }\end{array}$ & $\begin{array}{l}\text { Discharge } \\
\text { Mean (SD) }\end{array}$ & Mean difference & $95 \% \mathrm{Cls}^{*}$ & $\mathbf{t}$ & $\begin{array}{l}\text { p Value } \\
\text { Two-tailed }\end{array}$ \\
\hline \multicolumn{7}{|c|}{ Functional independence (FIM+FAM) } \\
\hline Self-care & $26.2(13.0)$ & $34.7(13.4)$ & 8.6 & 8.3 to 8.8 & 71.6 & $<0.001$ \\
\hline Sphincter & $7.2(4.8)$ & $9.7(4.8)$ & 2.5 & 2.4 to 2.6 & 50.3 & $<0.001$ \\
\hline Transfers & $10.8(8.1)$ & $17.7(9.2)$ & 7.0 & 6.7 to 7.1 & 72.6 & $<0.001$ \\
\hline Locomotion & $6.4(4.7)$ & $10.9(6.0)$ & 4.6 & 4.5 to 4.7 & 71.1 & $<0.001$ \\
\hline Communication & $21.9(10.2)$ & $26.1(9.2)$ & 4.2 & 4.1 to 4.4 & 54.4 & $<0.001$ \\
\hline Psychosocial & $16.2(7.4)$ & $19.9(6.9)$ & 3.7 & 3.5 to 4.8 & 54.4 & $<0.001$ \\
\hline Cognition & $19.8(10.4)$ & $24.7(9.6)$ & 5.0 & 4.8 to 5.1 & 57.6 & $<0.001$ \\
\hline \multicolumn{7}{|c|}{ Subscale and total scores FIM+FAM } \\
\hline Motor & $50.6(27.9)$ & $72.9(31.6)$ & 22.7 & 22.1 to 23.3 & 79.7 & $<0.001$ \\
\hline Cognitive & $58.0(26.0)$ & $70.8(24.2)$ & 12.8 & 12.5 to 13.3 & 64.6 & $<0.001$ \\
\hline Total FIM+FAM & $108.5(47.1)$ & $143.7(51.0)$ & 35.5 & 34.6 to 36.4 & 83.8 & $<0.001$ \\
\hline \multicolumn{7}{|c|}{ Subscale and total scores FIM only† } \\
\hline Motor & $41.5(24.2)$ & $59.9(26.7)$ & 18.4 & 17.9 to 18.8 & 76.7 & $<0.001$ \\
\hline Cognitive & $21.7(10.0)$ & $25.9(9.0)$ & 4.2 & 4.0 to 4.3 & 56.5 & $<0.001$ \\
\hline Total FIM & $63.1(30.2)$ & $85.8(33.1)$ & 22.6 & 22.1 to 23.1 & 80.5 & $<0.001$ \\
\hline \multicolumn{7}{|c|}{ Dependency (NPDS/NPCNA) } \\
\hline Total NPDS score & $31.0(17.4)$ & $20.8(17.6)$ & -10.3 & -10.7 to -10.0 & -59.6 & $<0.001$ \\
\hline Care hours/week & $44.7(19.5)$ & $31.7(21.2)$ & -13.0 & -13.4 to -12.6 & -59.2 & $<0.001$ \\
\hline Care costs/week & $£ 1580(£ 933)$ & $£ 1083(£ 950)$ & $-£ 496$ & $-£ 517$ to $-£ 475$ & -45.9 & $<0.001$ \\
\hline \multicolumn{7}{|c|}{ Cost-efficiency parameters } \\
\hline & & & Mean & $95 \% \mathrm{Cl}$ & & \\
\hline \multicolumn{3}{|l|}{ FIM efficiency } & 0.42 & 0.41 to 0.44 & & \\
\hline \multicolumn{3}{|l|}{ FIM+FAM efficiency } & 0.67 & 0.64 to 0.69 & & \\
\hline \multicolumn{3}{|c|}{ Time to offset the costs of rehabilitation (months) } & 17.9 & 14.5 to 21.4 & & \\
\hline
\end{tabular}

${ }^{*}$ Bootstrapped Cls based on 1000 bootstrap samples.

†FIM sores are provided for comparison with other series.

FIM, Functional Independence Measure; FIM+FAM, UK Functional Assessment Measure; NPDS, Northwick Park Dependency Score; NPCNA, Northwick Park Care Needs Assessment. 


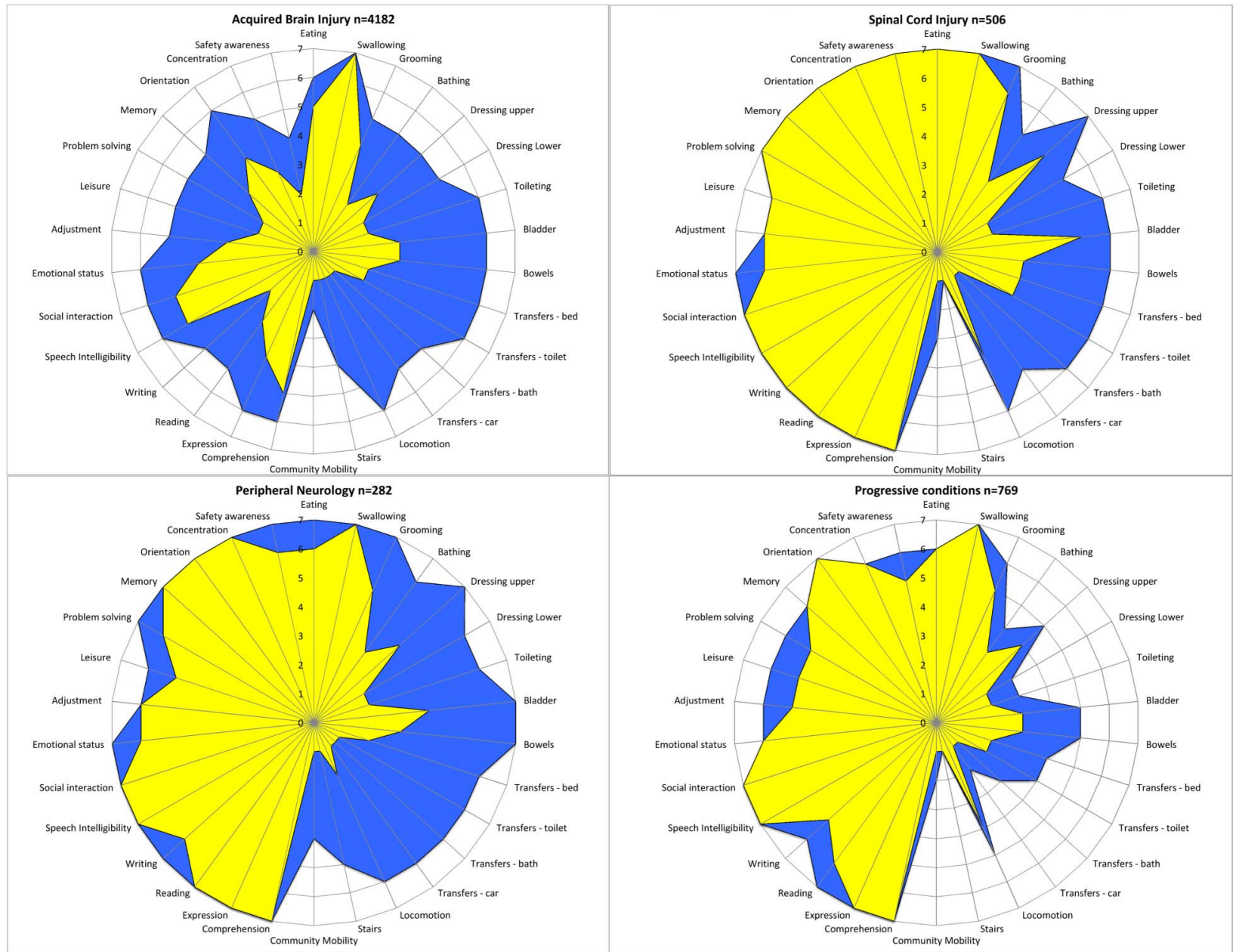

Figure 2 The radar chart (or 'FAM splat') provides a graphic representation of the disability profile from the FIM+FAM data. The 30-scale items are arranged as spokes of a wheel. Scoring levels from 1 (total dependence) to 7 (total independence) run from the centre outwards. Thus, a perfect score would be demonstrated as a large circle. This composite radar chart illustrates the median scores on admission and discharge. The yellow-shaded portion represents the median scores on admission for each item. The blue-shaded area represents the change in median score from admission to discharge. Clear differences in the pattern of disability can be seen between the four groups. FIM, Functional Independence Measure; FIM+FAM, UK Functional Assessment Measure.

the categories with the difference only crossing the threshold for significance between the ABI and SCI groups. However, as may be expected, cognitive FIM + FAM scores were significantly lower in ABI than all other diagnosis $(\mathrm{p}<0.001)$, and remained so at discharge despite the substantially greater change in this group (mean 15.7). Cognitive FIM+FAM scores were also significantly lower for progressive conditions than for the SCI and peripheral neurology groups, but the latter were similar.

Between admission and discharge, change in FIM +FAM motor score was significantly different between all groups $(p<0.001)$, except between ABI and SCI $(p=1.0)$. Change in FIM+FAM cognitive score was significantly different between all of the groups $(p<0.01)$ except for SCI and progressive conditions $(p=1.0)$. Mean FIM+FAM efficiency was lowest in progressive conditions (mean 0.44) followed by the SCI group (mean 0.59) while broadly similar in the ABI and peripheral neurology groups at a mean of 0.71 and 0.77 , respectively.

The differences in dependency are also summarised in table 3. In keeping with the above findings, the ABI group was the most dependent on admission. Post hoc tests showed NPDS and estimated weekly care costs to be significantly higher in ABI than all other groups $(\mathrm{p}<0.001)$, but there were no statistically significant differences between any of the other groups.

Between admission and discharge, reduction in dependency and care costs were significantly different between all groups $(p<0.001)$, except between $A B I$ and SCI $(p \geq 0.1)$. The mean individually calculated time to offset the cost of rehabilitation was lowest in the progressive conditions, at 8.5 months compared with 19-20 for the other groups, but the data were widely spread with overlapping CIs and post hoc tests did not show any significant between-group differences. 
Table 3 Comparison of functional and dependency scores between diagnostic groups

\begin{tabular}{|c|c|c|c|c|c|c|c|c|c|c|}
\hline \multirow[b]{2}{*}{ Parameter } & \multicolumn{2}{|c|}{ ABI $(n=4182)$} & \multicolumn{2}{|c|}{$\mathrm{SCl}(n=506)$} & \multicolumn{2}{|c|}{ Peripheral $(n=282)$} & \multicolumn{2}{|c|}{ Progressive $(n=769)$} & \multicolumn{2}{|c|}{ One-way ANOVA* } \\
\hline & Mean & $95 \% \mathrm{Cl}$ & Mean & $95 \% \mathrm{Cl}$ & Mean & $95 \% \mathrm{Cl}$ & Mean & $95 \% \mathrm{Cl}$ & $\overline{\mathbf{F}}$ & p Value \\
\hline UK FIM+FAM & & & & & & & & & Betwee & \\
\hline \multicolumn{11}{|l|}{ Admission } \\
\hline Motor & 50.1 & 49.2 to 51.0 & 57.1 & 55.2 to 58.9 & 51.2 & 48.6 to 54.0 & 52.8 & 51.0 to 54.4 & 11.2 & $<0.001$ \\
\hline Cognitive & 50.7 & 50.0 to 54.1 & 86.2 & 85.1 to 87.3 & 81.8 & 79.9 to 83.6 & 74.2 & 72.7 to 75.6 & 582.5 & $<0.001$ \\
\hline Total & 100.8 & 99.3 to 102.3 & 143.3 & 140.7 to 145.8 & 133.0 & 129.2 to 136.9 & 127.0 & 124.2 to 129.6 & 201.5 & $<0.001$ \\
\hline \multicolumn{11}{|l|}{ Discharge } \\
\hline Motor & 74.0 & 73.0 to 74.9 & 81.2 & 79.3 to 83.3 & 85.0 & 82.0 to 87.8 & 64.3 & 62.3 to 66.2 & 49.5 & $<0.001$ \\
\hline Cognitive & 66.4 & 65.6 to 67.1 & 90.7 & 88.9 to 91.5 & 90.3 & 89.0 to 91.5 & 78.9 & 77.6 to 80.3 & 255.3 & $<0.001$ \\
\hline Total & 140.4 & 138.7 to 141.9 & 171.9 & 169.5 to 174.5 & 175.3 & 171.6 to 178.9 & 143.2 & 140.2 to 146.0 & 91.8 & $<0.001$ \\
\hline \multicolumn{11}{|l|}{ Change } \\
\hline Motor & 23.9 & 23.2 to 24.5 & 24.1 & 22.5 to 25.7 & 33.8 & 31.2 to 36.7 & 11.5 & 10.5 to 12.5 & 97.7 & $<0.001$ \\
\hline Cognitive & 15.7 & 15.2 to 16.2 & 4.5 & 3.8 to 5.3 & 8.6 & 7.4 to 9.8 & 4.7 & 4.0 to 5.3 & 202.3 & $<0.001$ \\
\hline Total & 39.6 & 38.6 to 40.6 & 28.6 & 26.7 to 30.6 & 42.3 & 39.2 to 45.9 & 16.1 & 14.8 to 17.5 & 134.3 & $<0.001$ \\
\hline FIM efficiency & 0.44 & 0.42 to 0.46 & 0.43 & 0.39 to 0.47 & 0.54 & 0.49 to 0.61 & 0.29 & 0.26 to 0.33 & 22.0 & $<0.001$ \\
\hline FIM+FAM efficiency & 0.71 & 0.69 to 0.74 & 0.59 & 0.54 to 0.65 & 0.77 & 0.70 to 0.87 & 0.44 & 0.39 to 0.48 & 27.8 & $<0.001$ \\
\hline \multicolumn{11}{|l|}{ NPDS/NPCNA } \\
\hline \multicolumn{11}{|l|}{ Admission } \\
\hline NPDS total score & 32.2 & 31.7 to 32.8 & 24.2 & 23.0 to 25.4 & 27.7 & 26.0 to 29.4 & 26.6 & 25.5 to 27.7 & 49.0 & $<0.001$ \\
\hline Care hours/week & 45.4 & 44.9 to 46.0 & 39.8 & 38.2 to 41.3 & 44.6 & 42.2 to 4,67 & 43.1 & 41.6 to 44.5 & 13.9 & $<0.001$ \\
\hline Care costs & $£ 1667$ & $£ 1641$ to $£ 1695$ & $£ 1228$ & $£ 1152$ to $£ 1302$ & $£ 1452$ & $£ 1336$ to $£ 1561$ & $£ 1345$ & $£ 1278$ to $£ 1415$ & 46.6 & $<0.001$ \\
\hline \multicolumn{11}{|l|}{ Discharge } \\
\hline NPDS total score & 21.3 & 20.7 to 21.8 & 14.3 & 13.3 to 15.3 & 13.4 & 12.0 to 14.9 & 21.1 & 19.9 to 22.2 & 39.7 & $<0.001$ \\
\hline Care hours/week & 32.4 & 31.8 to 33.1 & 24.2 & 22.6 to 25.8 & 22.7 & 20.4 to 24.9 & 35.5 & 33.9 to 37.0 & 51.1 & $<0.001$ \\
\hline Care costs & $£ 1152$ & $£ 1123$ to $£ 1181$ & $£ 733$ & $£ 667$ to $£ 795$ & $£ 684$ & $£ 587$ to $£ 774$ & $£ 1057$ & $£ 986$ to $£ 1121$ & 40.6 & $<0.001$ \\
\hline \multicolumn{11}{|l|}{ Change } \\
\hline NPDS total score & -11.0 & -11.4 to -10.6 & -9.9 & -10.9 to -8.9 & -14.3 & -15.8 to -12.7 & -5.5 & -6.2 to -4.8 & 48.8 & $<0.001$ \\
\hline Care hours/week & -13.0 & -13.5 to -12.5 & -15.6 & -17.0 to -14.1 & -21.9 & -24.2 to -19.8 & -7.6 & -8.6 to -6.7 & 52.2 & $<0.001$ \\
\hline Care costs & $-£ 515$ & $-£ 541$ to $-£ 490$ & $-£ 495$ & $-£ 566$ to $£ 424$ & $-£ 767$ & $-£ 870$ to $£ 656$ & $-£ 289$ & -£342 to £237 & 25.3 & $<0.001$ \\
\hline $\begin{array}{l}\text { Time to offset costs of } \\
\text { rehabilitation (months) }\end{array}$ & 19.2 & 14.6 to 24.2 & 20.9 & 13.0 to 29.8 & 19.6 & 11.6 to 28.0 & 8.5 & 1.8 to 14.2 & 1.5 & 0.225 \\
\hline
\end{tabular}

${ }^{*}$ Bootstrap results based on 1000 bootstrap samples.

ABI, acquired brain injury; ANOVA, analysis of variance; FIM, Functional Independence Measure; FIM+FAM, UK Functional Assessment Measure; NPDS, Northwick Park Dependency Score;

NPCNA, Northwick Park Care Needs Assessment; SCI, spinal cord injury. 
Differences between groups based on dependency at admission

The change in dependency, care needs and cost of care in the community are summarised in table 4, grouped by the level of dependency on admission.

As anticipated, LOS and the total cost of the rehabilitation episode were greatest in the high-dependency group and smallest in the low-dependency group with some twofold difference between them, and post hoc tests showed significant differences seen between all three groups $(\mathrm{p}<0.001)$.

The ongoing care hours and costs of care in the community remained high at discharge in the same pattern as on admission, but the reduction in care hours and costs was greater in the higher dependency groups, reflecting the higher starting levels-again with significant differences between all dependency groups $(\mathrm{p}<0.001)$.

Despite the higher cost of the rehabilitation, the time to offset the costs of treatment through savings in the cost of ongoing community care was shortest in the high-dependency group at 14.2 months, followed by the medium-dependency group at 22.3 months, and longest in the low-dependency group 27.7 months. But, despite the nearly twofold difference between the means for the low-dependency and high-dependency groups, the CIs were wide and the between-group ANOVA only just reached significance at $\mathrm{p}=0.024$.

By contrast, FIM efficiency was highest in the mediumdependency group at 0.54 but similar between the lowdependency and high-dependency groups at 0.37 and 0.38 , respectively $(\mathrm{p}=0.15)$. FIM+FAM efficiency was also highest in the medium-dependency group at 0.83 , and again similar in the low-dependency and highdependency groups at 0.70 and 0.58 , respectively $(\mathrm{p}=0.65)$.

Because the data set was dominated by the ABI group, we also compared the main cost-efficiency parameters between dependency groups separately for each of the diagnostic groups-see table 5. A broadly similar pattern was seen in all the groups, with the time to offset the costs of rehabilitation being shortest in the highdependency group (albeit with wide CIs), while FIM efficiency tended to be highest in the medium-dependency group-reaching significance in all diagnostic groups except the peripheral neurological conditions.

\section{DISCUSSION}

Large cohort analyses of routinely collected outcome data make an important contribution to our understanding of the gains that can be made from rehabilitation in the course of real-life clinical practice, and provide the opportunity for comparing different populations and practices. This first multicentre analysis of the UK national clinical data set for specialist rehabilitation demonstrates that patients with complex neurological disability have the potential to gain from specialist rehabilitation across a wide range of conditions. It confirmed that the findings from the previous single-centre study of ABI patients ${ }^{21}$ were generalisable across multiple centres and a wider range of neurological conditions. Although the costs of treatment were quite high ( $\$ 40000$ on average), this investment was offset by savings in the cost of ongoing care with approximately 18 months.

It should be noted that 'specialist rehabilitation' means something rather different in the UK from other countries. In the USA and Australia, a 'specialist rehabilitation centre' would be one in which the central focus of treatment is rehabilitation, often in diagnosisspecific programmes (eg, head injury, stroke or spinal cord rehabilitation). In the UK, the term 'specialist rehabilitation' is reserved for tertiary (levels 1 and 2) centres, serving a large catchment population (typically 1-5 million for level 1 units) and admitting a selected population of patients with highly complex rehabilitation needs, regardless of diagnosis. ${ }^{16}$ Thus, a stroke unit that provides rehabilitation as part of a specialist stroke programme would be classed as a level 3 (nonspecialised) rehabilitation service. Patients who would progress satisfactorily within their local (level 3) rehabilitation services were not included in this analysis, which therefore represents a smaller subgroup of more complex patients, in comparison with other international rehabilitation cohorts. Our findings may nevertheless have relevance for other health systems that offer tertiary programmes of care.

The time since onset was highly skewed but, on average, very long (eg, 9 months in the ABI group) compared with other published series. ${ }^{27}$ Lengths of stay were also substantially longer compared with recently published series from the $\mathrm{USA}^{11-13}$ and Australia, ${ }^{26}$ so that FIM efficiency was comparatively lower (0.4 compared with $0.4-0.8$ in the Australian series and 1.9-2.2 in the US series). These findings reflect the selected group of patients with complex needs admitted to the levels 1 and 2 services, many of whom had already failed to progress in their local level 3 rehabilitation services. Direct comparison of casemix-adjusted outcomes between the UK and Australian data sets ${ }^{28}$ confirms the preponderance of very severely disabled patients in the UK series, especially in the level 1 services. The majority of units contributing to the US and Australian data sets would be more similar to levels $2 \mathrm{~b}$ and 3 services in the UK (Eagar K, personal communication, 2015).

Nevertheless, for a UK population with mean age 47 years in 2015, the average projected life expectancy would be approximately 40 years (males) and 42 years (females). ${ }^{29}$ Even if one allows an estimated 15-year reduction in respect of complex neurological disability, the mean life expectancy of this study group may be 25 years or more. Extrapolated over this period, the mean saving of nearly $£ 500$ per week (or $£ 26 \mathrm{~K}$ per year) in ongoing costs of care might be expected to lead to overall life-time economic gains in excess of 
Table 4 Comparison of costs and efficiency between dependency groups $(n=5739)$

\begin{tabular}{|c|c|c|c|c|c|c|c|c|}
\hline \multirow[b]{2}{*}{ Parameter } & \multicolumn{2}{|c|}{$\begin{array}{l}\text { Low dependency (admission } \\
\text { NPDS }<10) \\
n=699(12 \%)\end{array}$} & \multicolumn{2}{|c|}{$\begin{array}{l}\text { Medium dependency } \\
\text { (admission NPDS 10-24) } \\
\mathrm{n}=1607(28 \%)\end{array}$} & \multicolumn{2}{|c|}{$\begin{array}{l}\text { High dependency (admission } \\
\text { NPDS } \geq 25) \\
n=3433(60 \%)\end{array}$} & \multicolumn{2}{|c|}{ One-way ANOVA } \\
\hline & Mean & $95 \% \mathrm{Cl}$ & Mean & $95 \% \mathrm{Cl}$ & Mean & $95 \% \mathrm{Cl}$ & $\bar{F}$ & p Value \\
\hline Length of stay (days) & 51 & 47 to 54 & 62 & 59 to 64 & 102 & 99 to 104 & 376.3 & $<0.001$ \\
\hline Cost of rehabilitation & £23 997 & $\begin{array}{l}£ 22025 \text { to } \\
£ 26089\end{array}$ & $£ 28473$ & $\begin{array}{l}£ 27181 \\
\text { to } £ 29731\end{array}$ & $£ 47111$ & $\begin{array}{l}£ 45789 \text { to } \\
£ 448314\end{array}$ & 345.0 & $<0.001$ \\
\hline \multicolumn{9}{|l|}{ NPDS/NPCNA } \\
\hline \multicolumn{9}{|l|}{ Admission } \\
\hline NPDS total score & 5.6 & 5.4 to 5.8 & 17.2 & 16.9 to 17.4 & 41.7 & 41.3 to 42.1 & 5401.7 & $<0.001$ \\
\hline Care hours/week & 15.9 & 15.2 to 16.6 & 31.9 & 31.332 .4 & 57.1 & 56.6 to 57.5 & 4160.8 & $<0.001$ \\
\hline Care costs $£ /$ week & $£ 436$ & $£ 402$ to $£ 470$ & $£ 926$ & $£ 897$ to $£ 954$ & $£ 2109$ & $£ 2083$ to $£ 2136$ & 2466.9 & $<0.001$ \\
\hline \multicolumn{9}{|l|}{ Discharge } \\
\hline NPDS total score & 5.1 & 4.6 to 5.5 & 9.5 & 9.1 to 9.9 & 25.7 & 25.2 to 26.3 & 913.1 & $<0.001$ \\
\hline Care hours/week & 11.3 & 10.5 to 12.0 & 18.7 & 18.0 to 19.4 & 39.1 & 38.4 to 39.8 & 966.1 & $<0.001$ \\
\hline Care costs $£ /$ week & $£ 306$ & $£ 271$ to $£ 342$ & $£ 517$ & $-£ 436$ to $-£ 547$ & $£ 1349$ & $£ 1315$ to $£ 1384$ & 689.9 & $<0.001$ \\
\hline \multicolumn{9}{|l|}{ Change } \\
\hline NPDS total score & -0.5 & -1.0 to -0.0 & -7.6 & -8.0 to -7.2 & -16.0 & -16.5 to -15.5 & 468.0 & $<0.001$ \\
\hline Care hours/week & -4.6 & -5.5 to -3.8 & -13.2 & -13.9 to -12.5 & -18.0 & -18.7 to -17.3 & 157.4 & $<0.001$ \\
\hline Care costs $£ /$ week & $-£ 130$ & $£-178$ to $-£ 82$ & $-£ 408$ & $£-445$ to $-£ 370$ & $-£ 760$ & $£-794$ to $-£ 726$ & 174.2 & $<0.001$ \\
\hline \multicolumn{9}{|l|}{ Efficiency } \\
\hline $\begin{array}{l}\text { Time to offset costs of } \\
\text { rehabilitation (months) }\end{array}$ & 27.7 & 15.9 to 39.7 & 22.3 & 16.9 to 29.2 & 14.2 & 9.9 to 18.8 & 3.7 & $<0.024$ \\
\hline FIM efficiency & 0.37 & 0.34 to 0.41 & 0.54 & 0.51 to 0.56 & 0.38 & 0.37 to 0.40 & 51.4 & $<0.001$ \\
\hline FAM efficiency & 0.70 & 0.64 to 0.77 & 0.83 & 0.79 to 0.88 & 0.58 & 0.56 to 0.61 & 54.3 & $<0.001$ \\
\hline
\end{tabular}

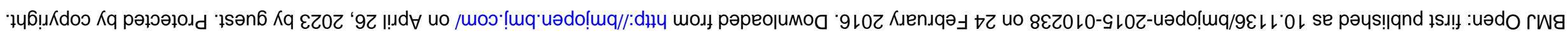


Table 5 Comparison of costs and cost-efficiency between dependency groups separated by diagnostic condition

\begin{tabular}{|c|c|c|c|c|c|c|c|c|}
\hline \multirow[b]{2}{*}{ Parameter } & \multicolumn{2}{|c|}{$\begin{array}{l}\text { Low dependency (admission } \\
\text { NPDS < 10) }\end{array}$} & \multicolumn{2}{|c|}{$\begin{array}{l}\text { Medium dependency } \\
\text { (admission NPDS 10-24) }\end{array}$} & \multicolumn{2}{|c|}{$\begin{array}{l}\text { High dependency (admission } \\
\text { NPDS } \geq 25 \text { ) }\end{array}$} & \multicolumn{2}{|c|}{ One-way ANOVA } \\
\hline & Mean & $95 \% \mathrm{Cl}$ & Mean & $95 \% \mathrm{Cl}$ & Mean & $95 \% \mathrm{Cl}$ & $\overline{\mathbf{F}}$ & p Value \\
\hline $\mathrm{ABI}$ & $N=339$ & & $\mathrm{~N}=872$ & & $\mathrm{~N}=2113$ & & & \\
\hline Cost of rehabilitation episode & $£ 27360$ & $£ 24300$ to $£ 30305$ & $£ 30591$ & $£ 28842$ to $£ 32292$ & $£ 49986$ & $£ 48637$ to $£ 51406$ & 166.3 & $<0.001$ \\
\hline Reduction in weekly care costs & $£ 152$ & $£ 91$ to $£ 215$ & $£ 463$ & $£ 419$ to $£ 506$ & $£ 760$ & $£ 721$ to $£ 799$ & 102.9 & $<0.001$ \\
\hline Time to offset costs (months) & 28.8 & 13.1 to 46.3 & 25.6 & 17.0 to 36.9 & 15.0 & 9.6 to 20.6 & 2.9 & 0.06 \\
\hline FIM efficiency & 0.38 & 0.34 to 0.42 & 0.56 & 0.53 to 0.59 & 0.40 & 0.38 to 0.42 & 34.7 & $<0.001$ \\
\hline$\overline{\mathrm{SCl}}$ & $\mathrm{N}=58$ & & $\mathrm{~N}=169$ & & $\mathrm{~N}=\mathbf{2 1 0}$ & & & \\
\hline Cost of rehabilitation episode & $£ 18198$ & $£ 15179$ to $£ 21647$ & $£ 28204$ & $£ 24812$ to $£ 31442$ & $£ 43897$ & $£ 39825$ to $£ 48333$ & 30.9 & $<0.001$ \\
\hline Reduction in weekly care costs & $£ 45$ & $£ 95$ to $£ 177$ & $£ 407$ & $£ 407$ to $£ 511$ & $£ 847$ & $£ 772$ to $£ 973$ & 30.7 & $<0.001$ \\
\hline Time to offset costs (months) & 20.8 & $£ 9$ to $£ 58$ & 18.7 & 9.7 to 27.5 & 22.7 & 10.4 to 37.2 & 0.10 & 0.91 \\
\hline FIM efficiency & 0.37 & 0.28 to 0.46 & 0.55 & 0.46 to 0.63 & 0.36 & 0.31 to 0.41 & 8.4 & $<0.001$ \\
\hline Peripheral conditions & $\mathrm{N}=29$ & & $\mathrm{~N}=87$ & & $\mathrm{~N}=144$ & & & \\
\hline Cost of rehabilitation episode & $£ 20814$ & $£ 16539$ to $£ 26180$ & $£ 29491$ & $£ 24338$ to $£ 35255$ & $£ 45339$ & $£ 40021$ to $£ 51054$ & 11.9 & $<0.001$ \\
\hline Reduction in weekly care costs & $£ 227$ & $£ 79$ to $£ 409$ & $£ 405$ & $£ 260$ to $£ 555$ & $£ 1207$ & $£ 1049$ to $£ 1372$, & 32.1 & $<0.001$ \\
\hline Time to offset costs (months) & 42.7 & 11.0 to 70.8 & 17.8 & 12.1 to 24.6 & 16.1 & 2.9 to 28.8 & 1.9 & 0.154 \\
\hline FIM efficiency & 0.51 & 0.33 to 0.71 & 0.56 & 0.46 to 0.65 & 0.54 & 0.46 to 0.63 & 0.1 & 0.889 \\
\hline Progressive conditions & $\mathrm{N}=72$ & & $\mathrm{~N}=\mathbf{2 1 0}$ & & $\mathrm{N}=344$ & & & \\
\hline Cost of rehabilitation episode & $£ 14118$ & $£ 11828$ to $£ 16643$ & $£ 19476$ & $£ 17140$ to $£ 21975$ & $£ 31991$ & $£ 29269$ to $£ 34773$ & 33.8 & $<0.001$ \\
\hline Reduction in weekly care costs & $£ 54$ & $£ 30$ to $£ 142$ & $£ 182$ & $£ 94$ to $£ 266$ & $£ 520$ & $£ 427$ to $£ 616$ & 19.3 & $<0.001$ \\
\hline Time to offset costs (months) & 21.6 & 7.4 to 36.9 & 13.3 & 6.9 to 20.3 & 2.8 & -7.4 to 12.9 & 2.3 & 0.096 \\
\hline FIM efficiency & 0.31 & 0.24 to 0.39 & 0.43 & 0.36 to 0.51 & 0.20 & 0.17 to 0.23 & 21.5 & $<0.001$ \\
\hline
\end{tabular}


$£ 650000$ or more per patient, or $£ 3.7$ billon for the whole study sample. This confirms the value of investing in appropriate specialist rehabilitation services for this group of patients. It does of course assume that the gains in independence are maintained. Evidence from a multicentre evaluation of community-based follow-up reported stability of dependency (and in some cases, further improvement) over the first year following discharge from the nine specialist levels 1 and 2a rehabilitation services in London, ${ }^{30}$ suggesting that this assumption is valid-and possibly even conservative -on a population basis.

Our analysis also demonstrated that cost-efficiency measured in this way was highest in the most dependent group of patients. This not only confirms the results from our previous single-centre study in patients with $\mathrm{ABI},{ }^{21}$ but also demonstrates that the reproducibility of this finding across multiple centres and different neurological conditions. FIM efficiency, meanwhile, appeared to be greatest in the medium-dependency group. This once again underlines the floor and ceiling effects the FIM in this more complex patient group and the fact that a linear trajectory of recovery cannot be assumed, nor an equal weight of items for estimating the cost of care needs.

These findings are important because, in many countries, these highly dependent patients may be denied rehabilitation if they are not expected to make significant gains on a FIM score. Thus, they emphasise the need for a range of different measures, reflecting different patient groups and their potential for change in during rehabilitation. FIM+FAM efficiency showed a similar pattern to FIM efficiency, so the additional 12 items did not necessarily improve its performance as a proxy for cost-efficiency, but they did provide a more holistic evaluation of the change in cognitive/psychosocial function, in addition to motor function, as illustrated in figure 2.

The authors recognise the following limitations to this study:

- The data were collected in the course of routine clinical practice. Despite the training provided to all units registered with UKROC, the exact level of expertise of clinicians recording the tools in each of the 62 centres is unknown. Nevertheless, the data set is reflective of real-life clinical practice, where staff experience is expected to vary.

- Because of the evolution of reporting requirements over the data collection period, the analysed sample represents less than $50 \%$ of the full rehabilitation data set. This finding was expected and comparison of demographic and baseline data suggested that the analysed sample was reasonably representative of the total population. Nevertheless, the possibility of selection bias cannot be excluded.

- The NPCNA estimates of continuing care costs are not true assessments as applied in traditional health economic studies. On the other hand, the instrument has been in use for over 15 years and is now quite widely taken up both in clinical practice and in research $^{24}$ Experience has demonstrated it to be neither overly generous nor mean in its estimation of care needs and costs. Moreover, for the purpose of this study, we were more interested in the relative values for between-group comparison than the absolute values. Nevertheless, the estimations of costsavings should be interpreted with some caution.

- Finally, while rehabilitation is provided through the health sector, the saving in care costs accrues to those responsible for ongoing care (typically the social care services or the patient and their family). Thus, the actual opportunity for realisation and reinvestment of the savings will depend on the local funding arrangements for health and social care.

The above limitations accepted, findings from this study add to the growing body of evidence for the costeffectiveness of rehabilitation for patients with complex disabilities. ${ }^{31} 32$ They confirm the potential for substantial cost-savings to be made from appropriate provision of specialist rehabilitation services for patients with complex needs, even many months after the original injury.

Acknowledgements The authors gratefully acknowledge the hard work of the clinical teams in the 62 centres, and of the patients and their families who participated in the follow-up survey. Special thanks are due to the UKROC programme steering group and co-applicants, and to Professor Kathy Eagar and colleagues in the Australasian Rehabilitation Outcome Centre (AROC) for information about the Australian database.

Contributors LT-S was the principal investigator for the larger programme of which this formed a part and leads the development of the UKROC register and data set. She took the lead role in planning, design, execution and governance of the study and had overall responsibility for study reporting. She performed the analyses presented within this article, and was responsible for drafting and submission of the manuscript. HW has played a lead role in development of the UKROC tools and outcome measurement. She is responsible for training of the teams who collect the data on the ground, and for data quality and checking. $A B$ is responsible for collating and analysing the data to signpost services to the relevant level; for gathering and calculation of episode cost data, and for data-checking and quality of this aspect of the project. PB specialises in providing statistical support for medical research and clinical trials. He provided overarching advice/support for the statistic analysis framework within the UKROC programme as well as specific advice for the techniques used in this article. KS is the UKROC data manager. He is responsible for programming, data management, information governance and data extraction. All authors were members of project steering group, contributed to writing the article, and to reviewing and commenting on the manuscript.

Funding This article presents independent research funded by the National Institute for Health Research (NIHR) under its Programme Grants for Applied Research programme (RP-PG-0407-10 185). Financial support for the preparation of this manuscript was also provided by the Dunhill Medical Trust.

Disclaimer The views expressed in this article are those of the authors and not necessarily those of the NHS, the NIHR or the Department of Health.

Competing interests Outcome measurement is a specific research interest of our centre. LT-S is Director of UKROC and was the lead developer of the NPDS, NPCNA and the UK FIM+FAM. All authors are employed by Northwick Park Hospital and/or King's College London, which may cite this article as part of their research evaluation processes, including the UK Research Excellence Framework 2020. LT-S, HW and KS have received financial support 
from the NIHR to attend conferences to disseminate the findings from the UK programme.

Ethics approval The UKROC programme is registered as a Multicentre Service Evaluation and as a Payment by Results Improvement Project. Collection and reporting of the UKROC data set is a commissioning requirement according to the NHSE service specification for levels 1 and 2 Rehabilitation Services.

Disclaimer According to the UK Health Research Authority, the publication of research findings from de-identified data gathered in the course of routine clinical practice does not require research ethics permission. Registration: the programme is registered with the NIHR Comprehensive Local Research Network: ID number 6352

Provenance and peer review Not commissioned; externally peer reviewed.

Data sharing statement No additional data are available.

Open Access This is an Open Access article distributed in accordance with the terms of the Creative Commons Attribution (CC BY 4.0) license, which permits others to distribute, remix, adapt and build upon this work, for commercial use, provided the original work is properly cited. See: http:// creativecommons.org/licenses/by/4.0/

\section{REFERENCES}

1. Neuro numbers - a brief review of the numbers of people in the UK with a neurological condition. London: The Neurological Alliance, 2003.

2. Khan F, Turner-Stokes L, Ng L, et al. Multidisciplinary rehabilitation for adults with multiple sclerosis. Cochrane Database Syst Rev 2007;8:CD006036.

3. Turner-Stokes L, Nair A, Disler PB, et al. Multi-disciplinary rehabilitation for acquired brain injury in adults of working age. Cochrane Database Syst Rev 2005;(3):CD004170.

4. DeJong G, Horn SD, Conroy B, et al. Opening the black box of post-stroke rehabilitation: stroke rehabilitation patients, processes, and outcomes. Arch Phys Med Rehabil 2005;86(12 Suppl 2):S1-S7

5. Horn SD, Gassaway J. Practice-based evidence study design for comparative effectiveness research. Med Care 2007;45(10 Suppl 2): S50-7.

6. The National Service Framework for Long Term Conditions. London: Department of Health, 2005.

7. Porter M, Teisberg E. Redefining health care: creating value-based competition on results. Boston: Harvard Business Press, 2006.

8. Bottemiller KL, Bieber PL, Basford JR, et al. FIM score, FIM efficiency, and discharge disposition following inpatient stroke rehabilitation. Rehabil Nurs 2006;31:22-5.

9. Khan F, Amatya B, Stevermuer T, et al. Rehabilitation for Guillain Barre syndrome: analysis of the Australian rehabilitation outcomes dataset. J Clin Med Res 2010;2:91-7.

10. Khan F, Turner-Stokes L, Stevermuer T, et al. Multiple sclerosis rehabilitation outcomes: analysis of a national casemix data set from Australia. Mult Scler 2009;15:869-75.

11. Granger CV, Markello SJ, Graham JE, et al. The uniform data system for medical rehabilitation: report of patients with stroke discharged from comprehensive medical programs in 2000-2007. Am J Phys Med Rehabil 2009;88:961-72.

12. Granger CV, Markello SJ, Graham JE, et al. The uniform data system for medical rehabilitation: report of patients with traumatic brain injury discharged from rehabilitation programs in 2000-2007. Am J Phys Med Rehabil 2010;89:265-78.
13. Granger CV, Karmarkar AM, Graham JE, et al. The uniform data system for medical rehabilitation: report of patients with traumatic spinal cord injury discharged from rehabilitation programs in 2002 2010. Am J Phys Med Rehabil 2012:91:289-99.

14. Hall KM, Mann N, High WMJ, et al. Functional measures after traumatic brain injury: ceiling effects of FIM, FIM+FAM, DRS, and CIQ. J Head Trauma Rehabil 1996;11:27-39.

15. Guide to the healthcare system in England. London: Department of Health, 2013.

16. Specialist neuro-rehabilitation services: providing for patients with complex rehabilitation needs. London: British Society of Rehabilitation Medicine, 2015.

17. Turner-Stokes L, Nyein K, Turner-Stokes T, et al. The UK FIM+FAM: development and evaluation. Clin Rehabil 1999;13:277-87.

18. Turner-Stokes L, Siegert RJ. A comprehensive psychometric evaluation of the UK FIM + FAM. Disabil Rehabil 2013;35:1885-95.

19. Turner-Stokes L, Tonge P, Nyein K, et al. The Northwick Park Dependency Score (NPDS): a measure of nursing dependency in rehabilitation. Clin Rehabil 1998;12:304-18.

20. Turner-Stokes L, Nyein K, Halliwell D. The Northwick Park Care Needs Assessment (NPCNA): a directly costable outcome measure in rehabilitation. Clin Rehabil 1999;13:253-67.

21. Turner-Stokes L, Paul S, Williams H. Efficiency of specialist rehabilitation in reducing dependency and costs of continuing care for adults with complex acquired brain injuries. J Neurol Neurosurg Psychiatr 2006;77:634-9.

22. Turner-Stokes L. Cost-efficiency of longer-stay rehabilitation programmes: can they provide value for money? Brain Inj 2007;21:1015-21.

23. Cost-efficient service provision in neurorehabilitation: defining needs, costs and outcomes for people with long term neurological conditions (RP-PG-0407-10185). Lead applicant: Professor Lynne Turner-Stokes. Northwick Park Hospital. London: National Institute for Health Research Programme Grant for Applied Research, 2008.

24. Siegert RJ, Turner-Stokes L. Psychometric evaluation of the Northwick Park Dependency Scale. J Rehabil Med 2010;42:936-43.

25. Turner-Stokes L, Sutch S, Dredge R. Healthcare tariffs for specialist inpatient neurorehabilitation services: rationale and development of a UK casemix and costing methodology. Clin Rehabil 2012;26:264-79.

26. Turner-Stokes L, Vanderstay R, Stevermuer T, et al. Comparison of rehabilitation outcomes for long term neurological conditions: a cohort analysis of the Australian Rehabilitation Outcomes Centre Dataset for Adults of Working Age. PLoS ONE 2015;10:e0132275.

27. Stineman MG, Ross RN, Hamilton BB, et al. Inpatient rehabilitation after stroke: a comparison of lengths of stay and outcomes in the Veterans affairs and non-Veterans affairs health care system. Med Care 2001;39:123-37.

28. Turner-Stokes L, Vanderstay R, Eagar K, et al. Cost-efficient service provision in neurorehabilitation: defining needs, costs and outcomes for people with long-term neurological conditions: programme grant report (RP-PG-0407-10185). London: National Institute of Health Research, 2015

29. Office for National Statistics. 2012-based period and cohort life expectancy tables. Fareham: UK Government ONS, 2014.

30. Siegert RJ, Jackson DM, Playford ED, et al. A longitudinal, multicentre, cohort study of community rehabilitation service delivery in long-term neurological conditions. BMJ Open 2014;4: e004231.

31. Turner-Stokes $L$. The evidence for the cost-effectiveness of rehabilitation following acquired brain injury. Clin Med 2004;4:10-12.

32. Oddy M, da Silva Ramos S. The clinical and cost-benefits of investing in neurobehavioural rehabilitation: a multi-centre study. Brain Inj 2013;27:1500-7. 